Supporting Information

\title{
Fluorescence Turn-On Response Amplified by Space Confinement in Metal-Organic Frameworks
}

Xue-Mei Yin, Lu-Lu Gao, Peng Li, Ran Bu, Weng-Jie Sun and En-Qing Gao*

Shanghai Key Laboratory of Green Chemistry and Chemical Processes, School of Chemistry and Molecular Engineering, East China Normal University, Shanghai 200062 (P. R. China).

*corresponding author. Email: eqgao@chem.ecnu.edu.cn 


\section{Physical Measurements}

${ }^{1} \mathrm{H}$ NMR spectra were recorded on a Bruker Advance $400 \mathrm{MHz}$ spectrometer. The FT-IR spectra were recorded in the range $500-4000 \mathrm{~cm}^{-1}$ using $\mathrm{KBr}$ pellets on a Nicolet NEXUS 670 spectrophotometer. Powder X-ray diffraction (PXRD) at ambient pressure was recorded on a Rigaku D/Max-2500 diffractometer at $35 \mathrm{kV}, 25 \mathrm{~mA}$ for a Cu-target tube and a graphite monochromator. UV-vis diffuse reflectance spectra were measured using a SHIMADZU UV-2700 spectrophotometer, with $\mathrm{BaSO}_{4}$ plates as references (100\% reflection). Liquid phase fluorescence spectra were recorded on a Hitachi F-4500 spectrofluorometer. Solid fluorescence spectra were obtained using a SHIMADZU RF-6000 spectrofluorometer. Scanning electron microscopy (SEM) was performed on a Hitachi S-4800 microscope. Nitrogen adsorption and desorption isotherm measurements were performed on a Micromeritics ASAP2020 analyzer at 77K. Thermal gravimetric analysis (TGA) was performed on a STA 449 F3 Simultaneous Thermal Analyzer in flowing air at $10^{\circ} \mathrm{C} / \mathrm{min}$. 


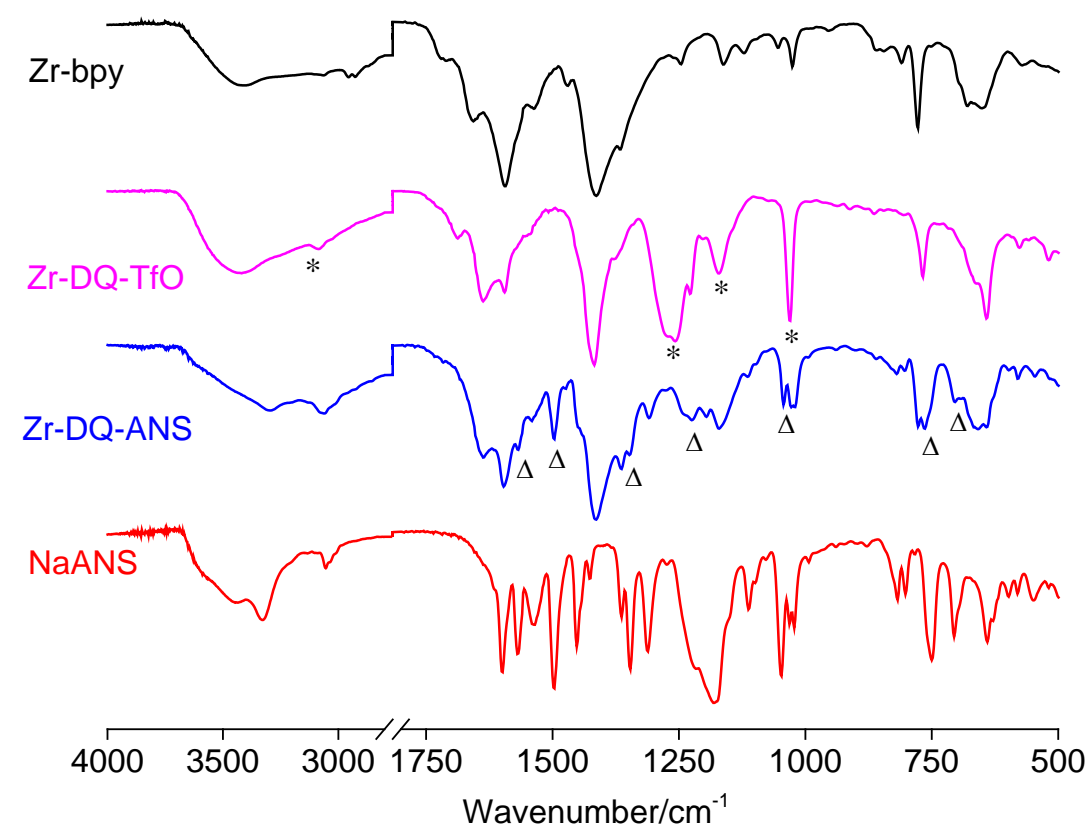

Figure S1. IR spectra of NaANS,, Zr-bpy, Zr-DQ-TfO, and Zr-DQ-ANS. The bands marked with * are new bands of Zr-DQ-TfO compared with Zr-bpy, and those marked with $\Delta$ are the bands arising from the ANS ${ }^{-}$anion, assigned in comparison with the spectrum of NaANS.

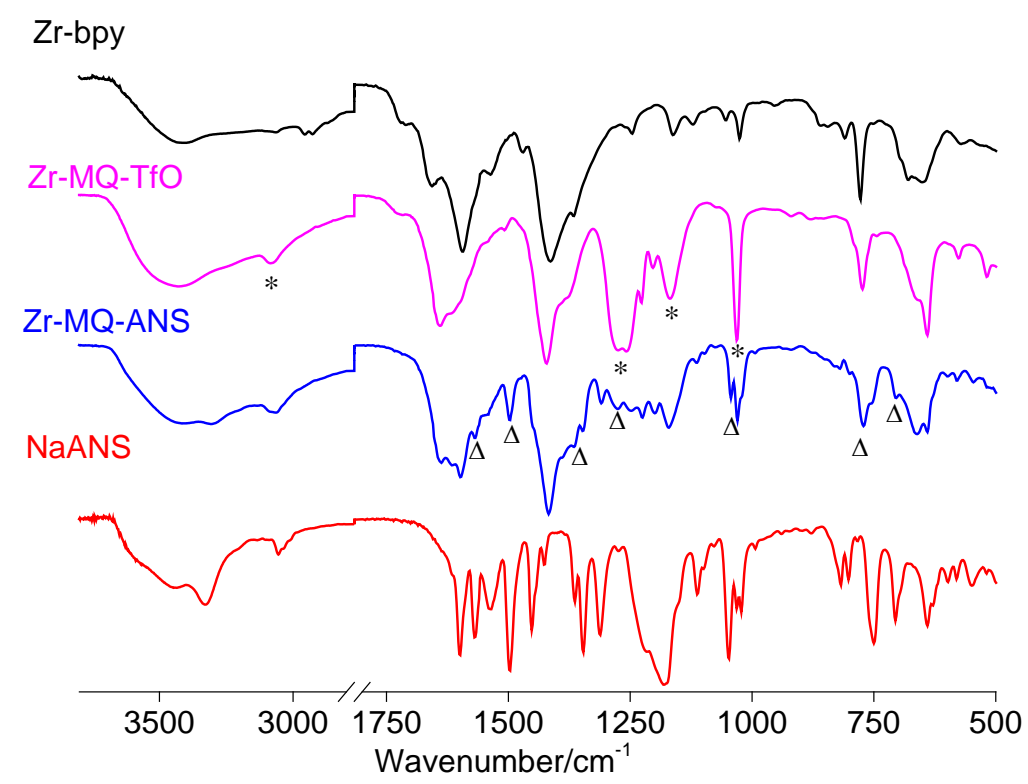

Figure S2. IR spectra of NaANS, Zr-bpy, Zr-MQ-TfO, and Zr-MQ-ANS. The bands marked with * are new bands of Zr-MQ-TfO compared with Zr-bpy, and those marked with $\Delta$ are the bands arising from the ANS ${ }^{-}$anion, assigned in comparison with the spectrum of NaANS. 


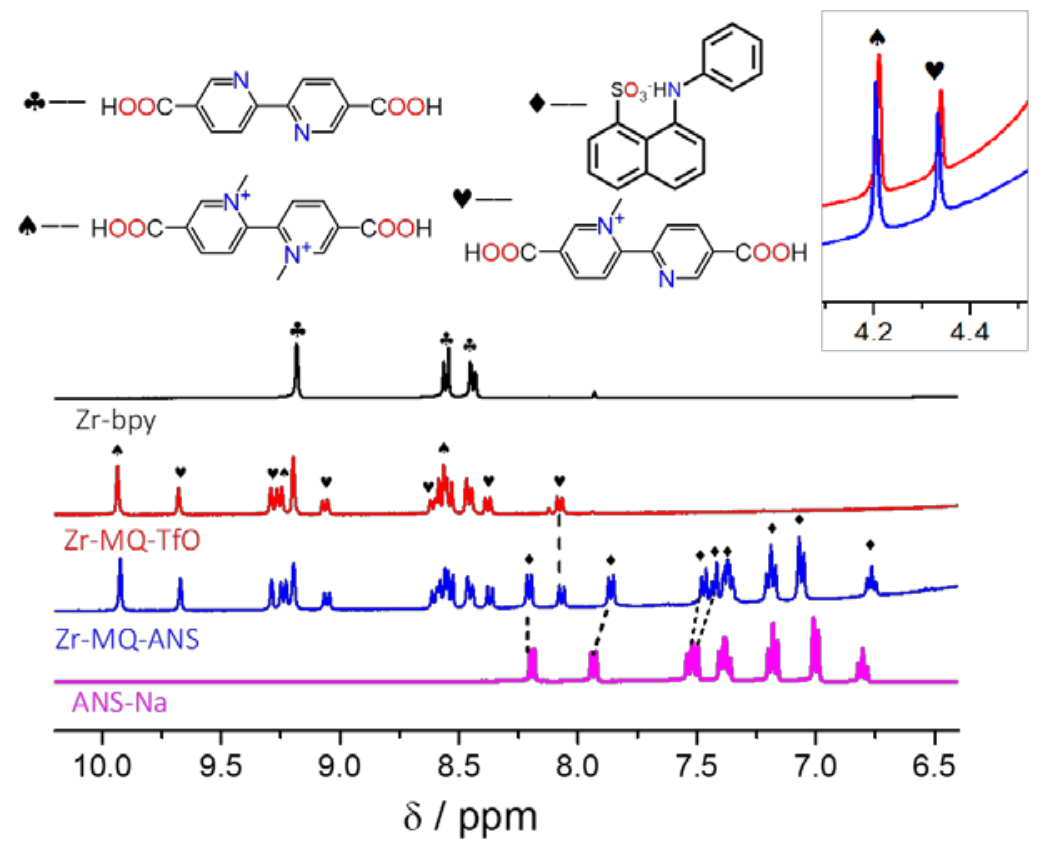

Figure S3. ${ }^{1} \mathrm{H}$ NMR spectra of Zr-DQ-TfO (magenta, N-alkylation ratio: $43 \%$ ) and Zr-DQ-ANS (blue). The spectra were recorded with the solutions obtained by digesting the solids with HF (aq.)/d 6 -DMSO (1/40, v/v).
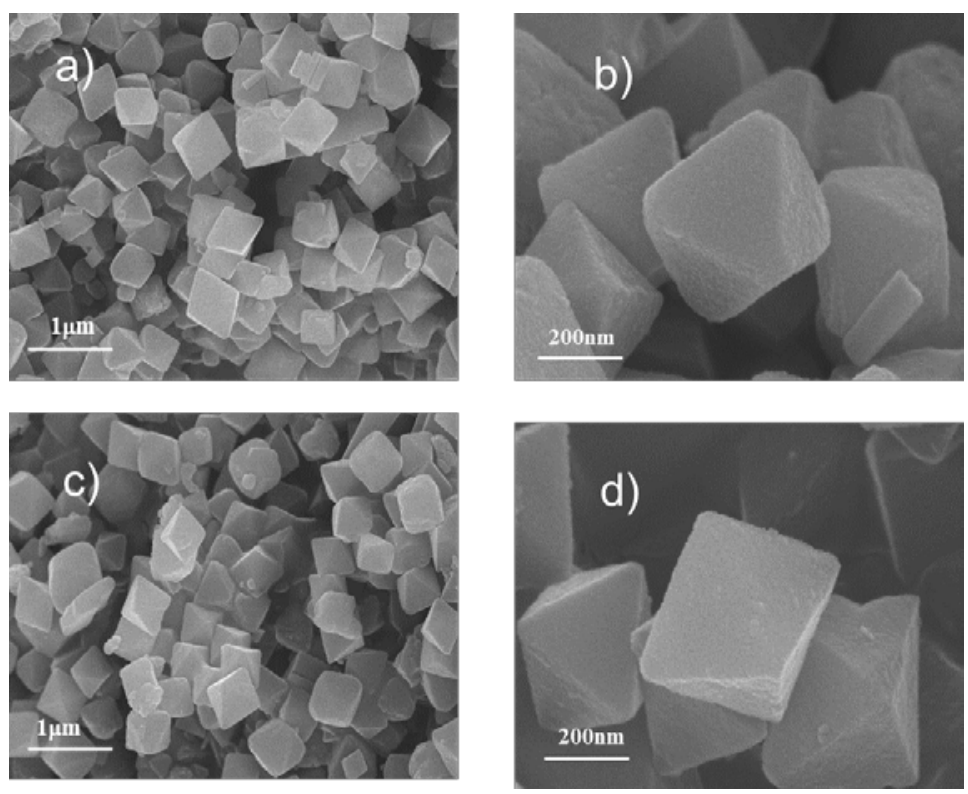

Figure S4. SEM images of Zr-MQ-TfO (a, b) and Zr-MQ-ANS (c, d). 


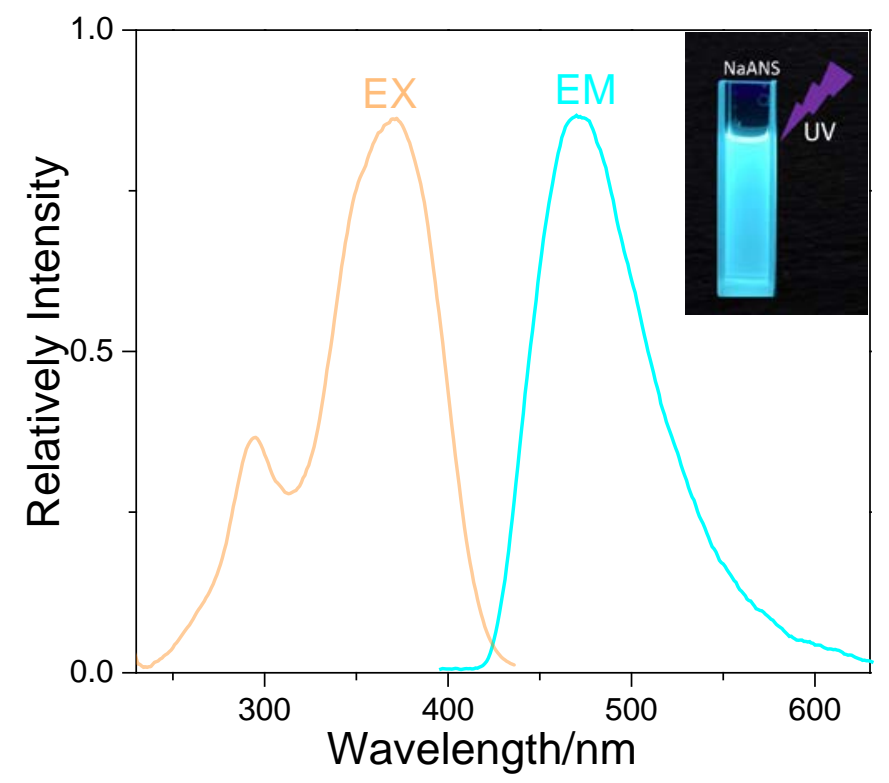

Figure S5. The excitation and emission spectra of NaANS in 1,4-dioxane $(0.1 \mu \mathrm{M})$.

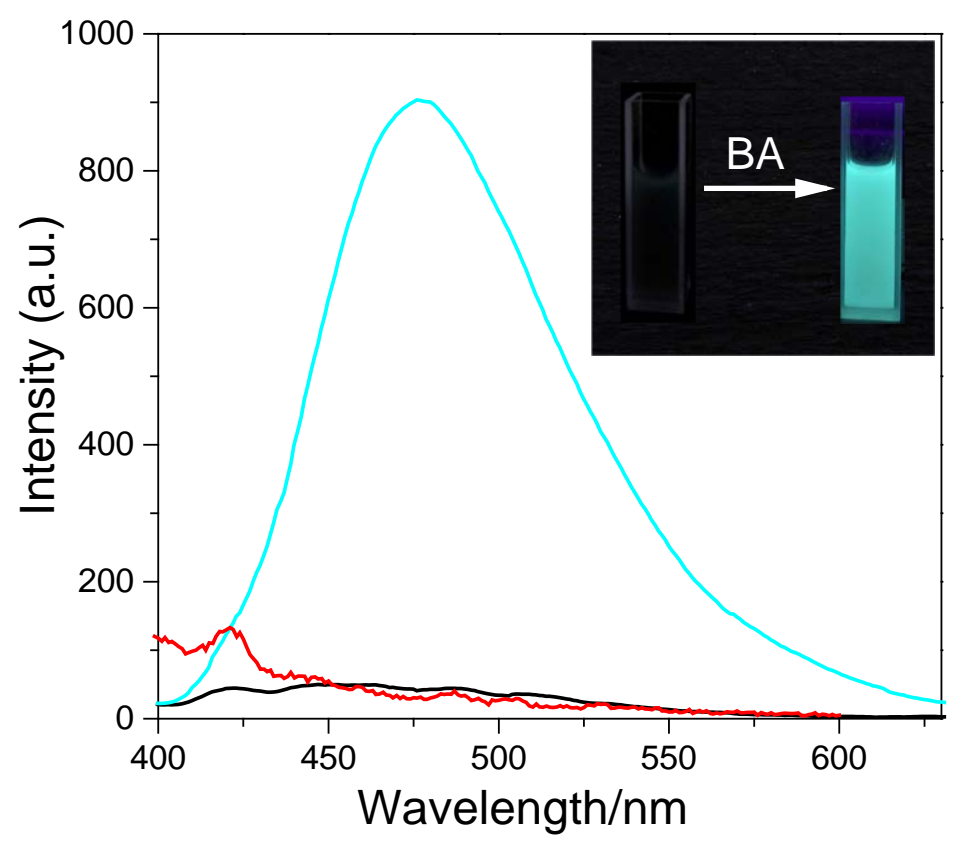

Figure S6. Emission spectra of Zr-MQ-TfO (red), Zr-MQ-ANS (black) dispersions in 1,4-dioxane and that of the Zr-MQ-ANS dispersion after addition of butylamine (BA, $10 \mu \mathrm{M})$ (cyan). The inset displays the BA-induced fluorescence turn-on of the Zr-MQ-ANS suspension under UV light (365 nm). 


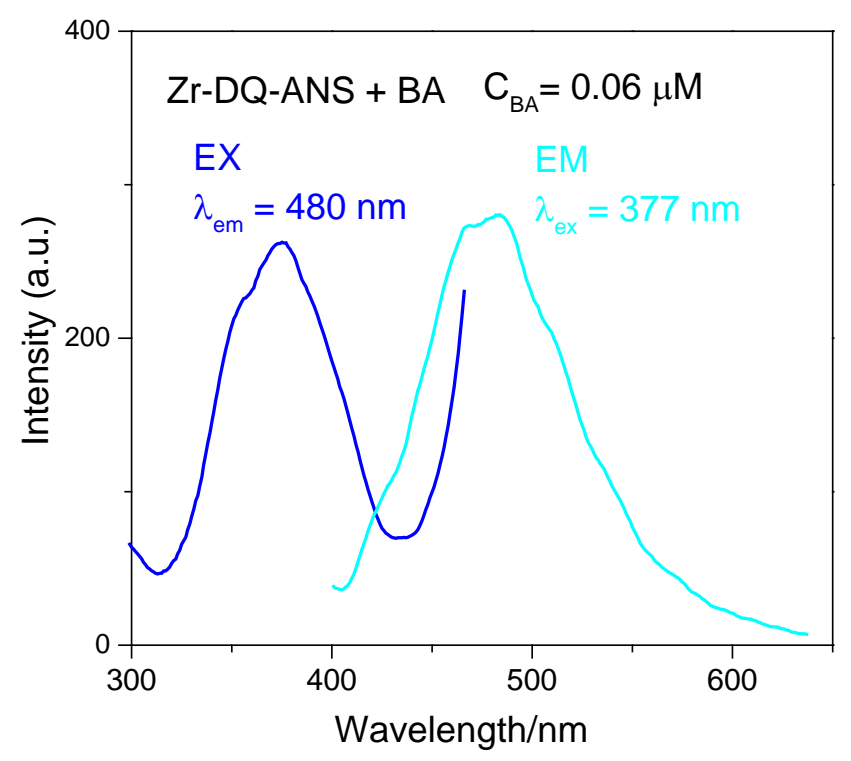

Figure S7. The excitation (blue) and emission spectra (cyan) of Zr-DQ-ANS. (CBA=0.06uM).
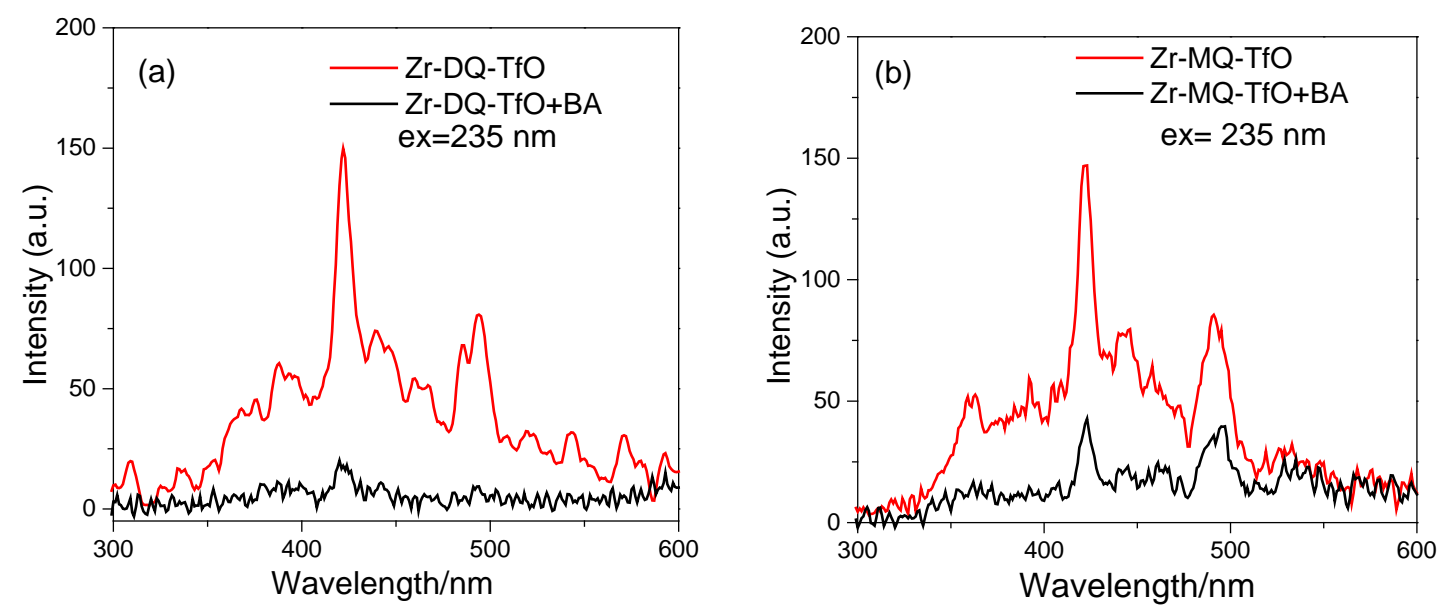

Figure S8. The emission spectra of Zr-DQ-TfO (a) and Zr-MQ-TfO (b) suspensions before and after addition of BA (0.1 M). 


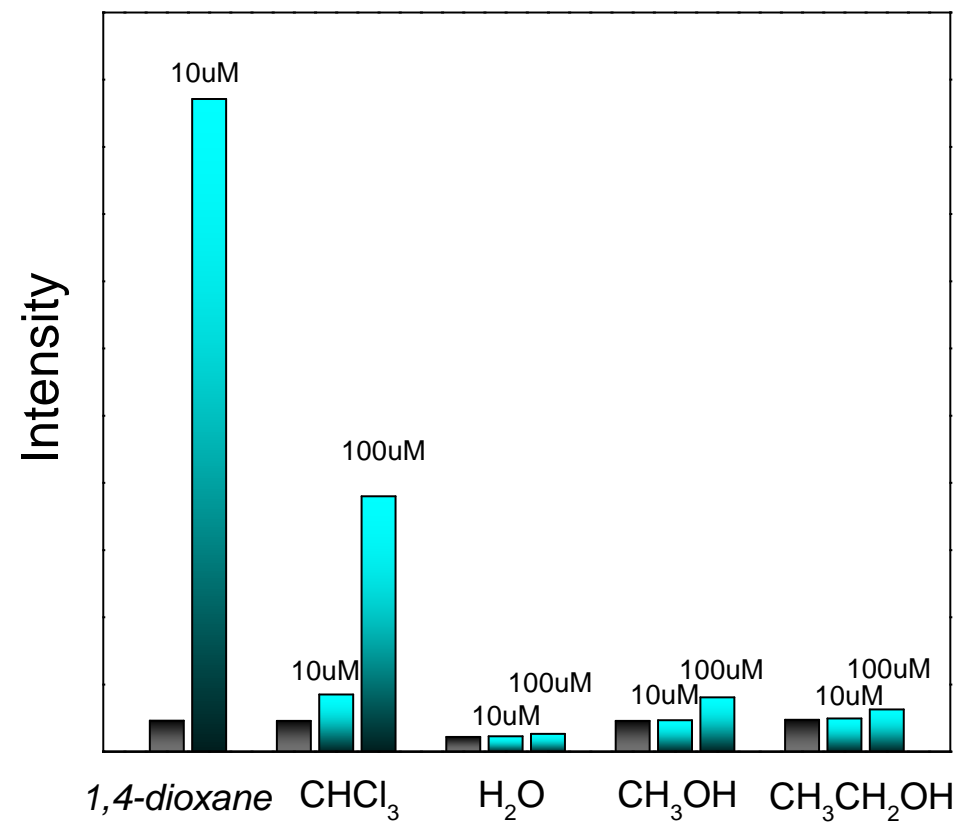

Figure S9. Luminescence intensities of Zr-DQ-ANS suspensions in different solvents in the absence (grey) and presence (cyan) of BA at the specified concentrations.

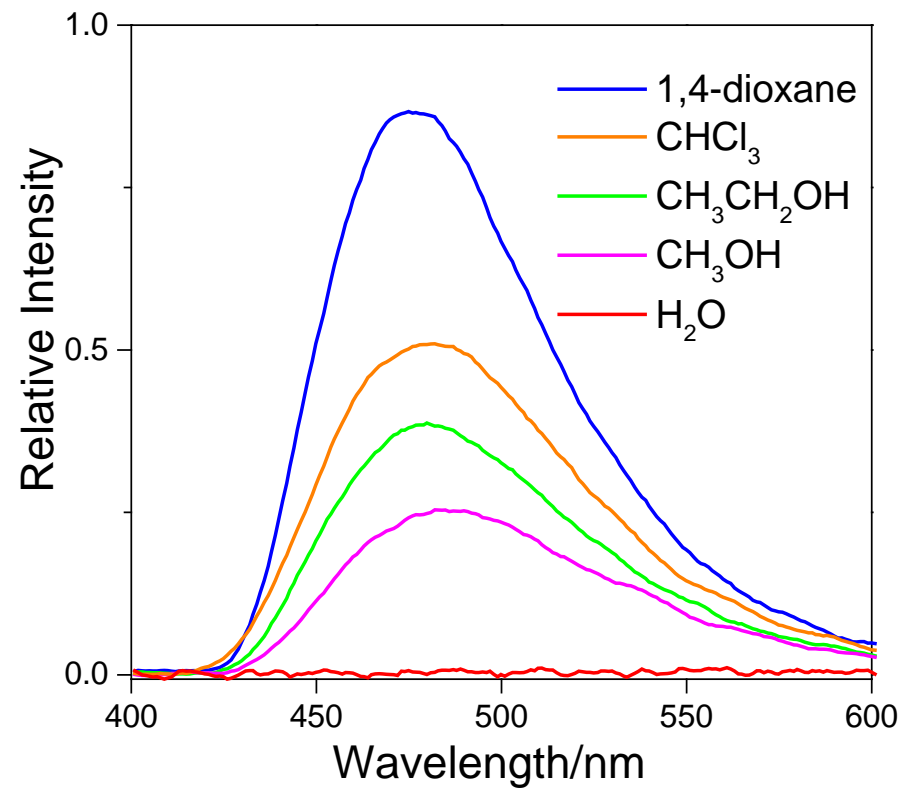

Figure S10. The emission spectra of NaANS in different solvents $\left(\lambda_{\mathrm{ex}}=377 \mathrm{~nm}\right)$. 


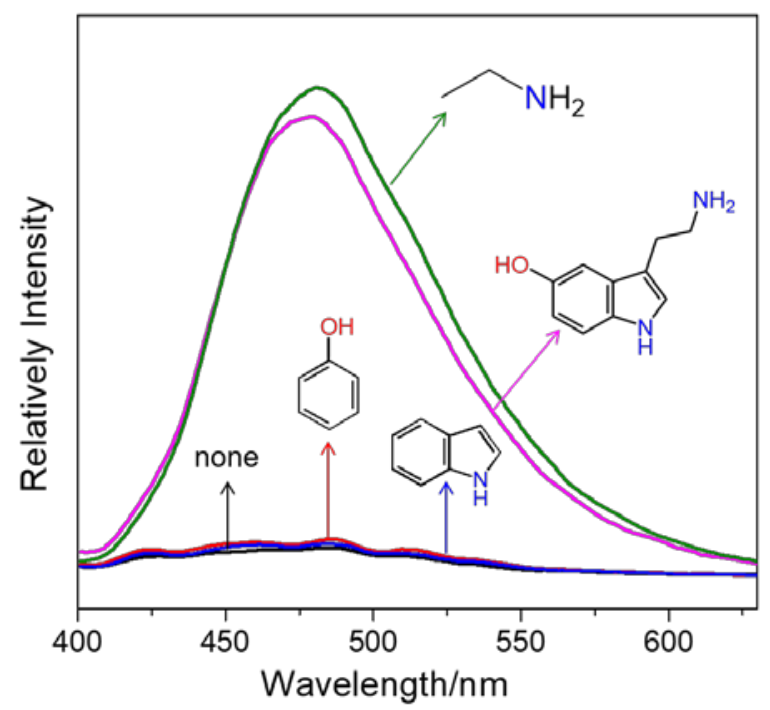

Figure S11. Emission spectra of the Zr-DQ-ANS dispersions in response to phenol, indole ethylamine and 5-HT (10 $\mu \mathrm{M})$.

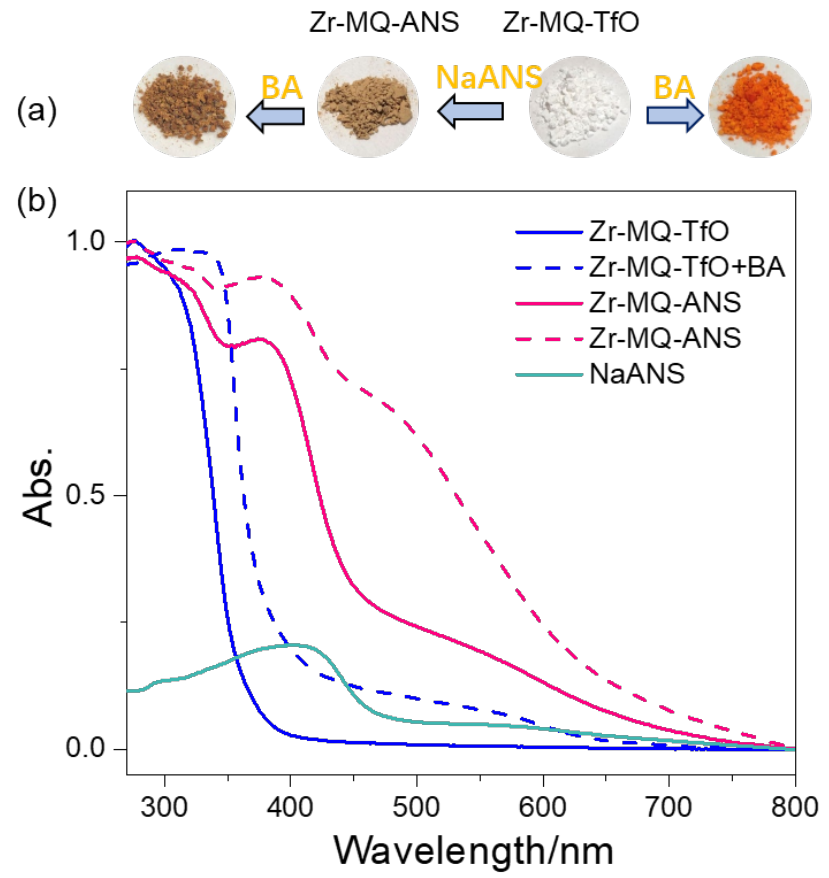

Figure S12. (a) Photographs showing the color changes before and after treating Zr-DQ-TfO and Zr-DQ-ANS with pure BA. (b) Normalized solid-state UV-vis spectra of Zr-DQ-TfO and Zr-DQ-ANS before (solid lines) and after (dashed lines) BA treatment. For comparison, the spectrum of NaANS is included. 


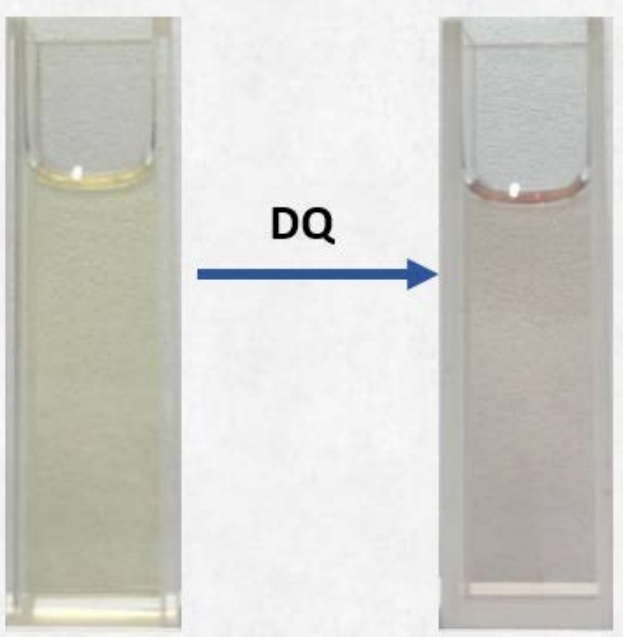

Figure S13. Photograph of color change before and after addition of $\left[\mathrm{Me}_{2} \mathrm{DQdc}\right]^{2+}$ to $\mathrm{NaANS}$ 1,4-dioxane solution.

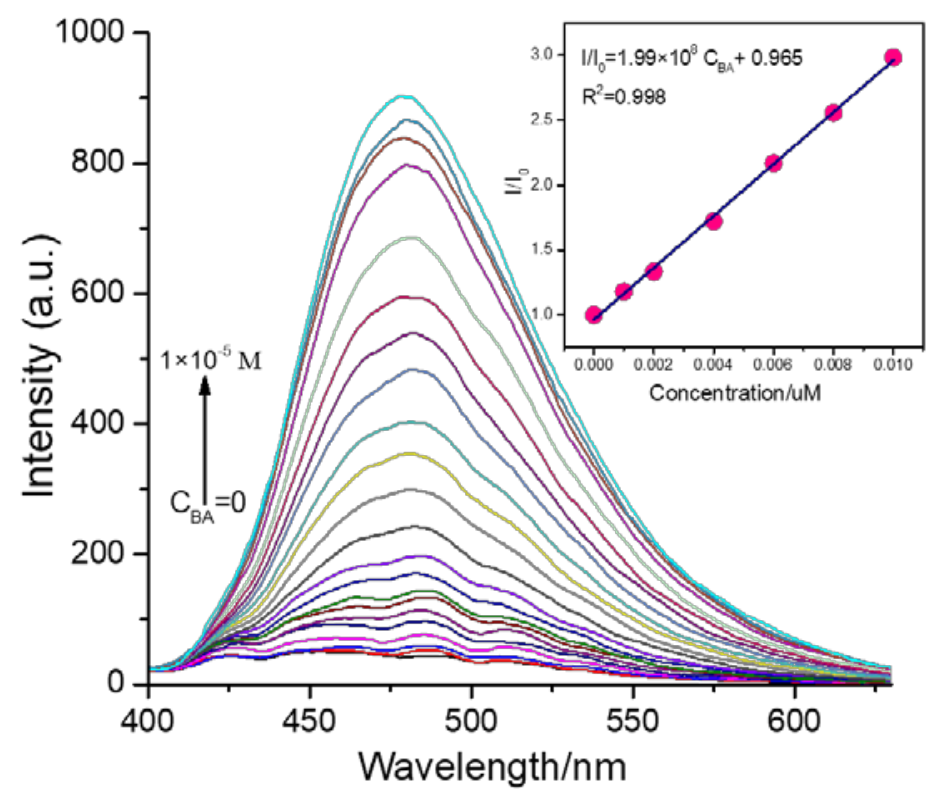

Figure S14. Emission spectra of the Zr-MQ-ANS dispersion in presence of different BA concentrations $\left(\lambda_{\mathrm{ex}}=377 \mathrm{~nm}\right)$. Inset: linear variation of $I / I_{0}($ at $480 \mathrm{~nm}$ ) with BA concentration in the low concentration region. 

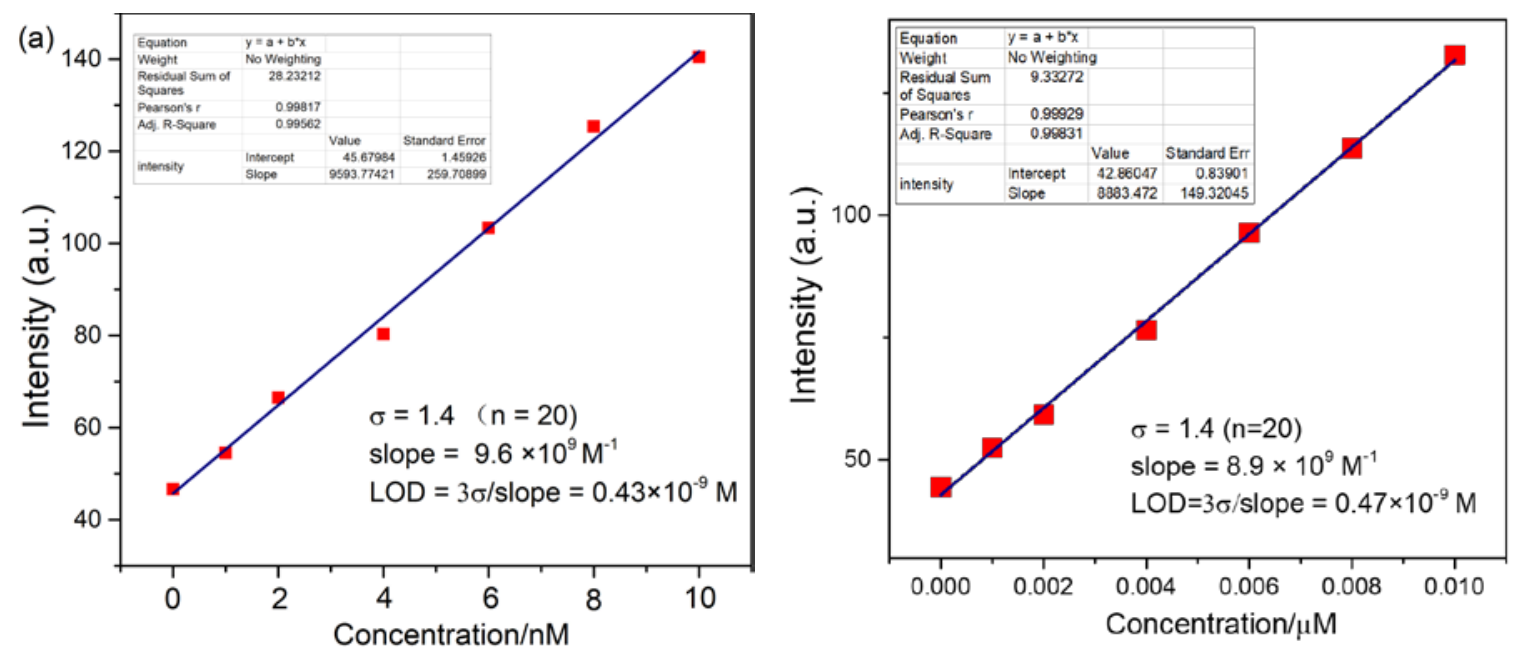

Figure S15. Data for calculations of LODs. The linear fit of the intensity-concentration data in low butylamine concentration range is shown. Left: Zr-DQ-ANS. Right: Zr-MQ-ANS.

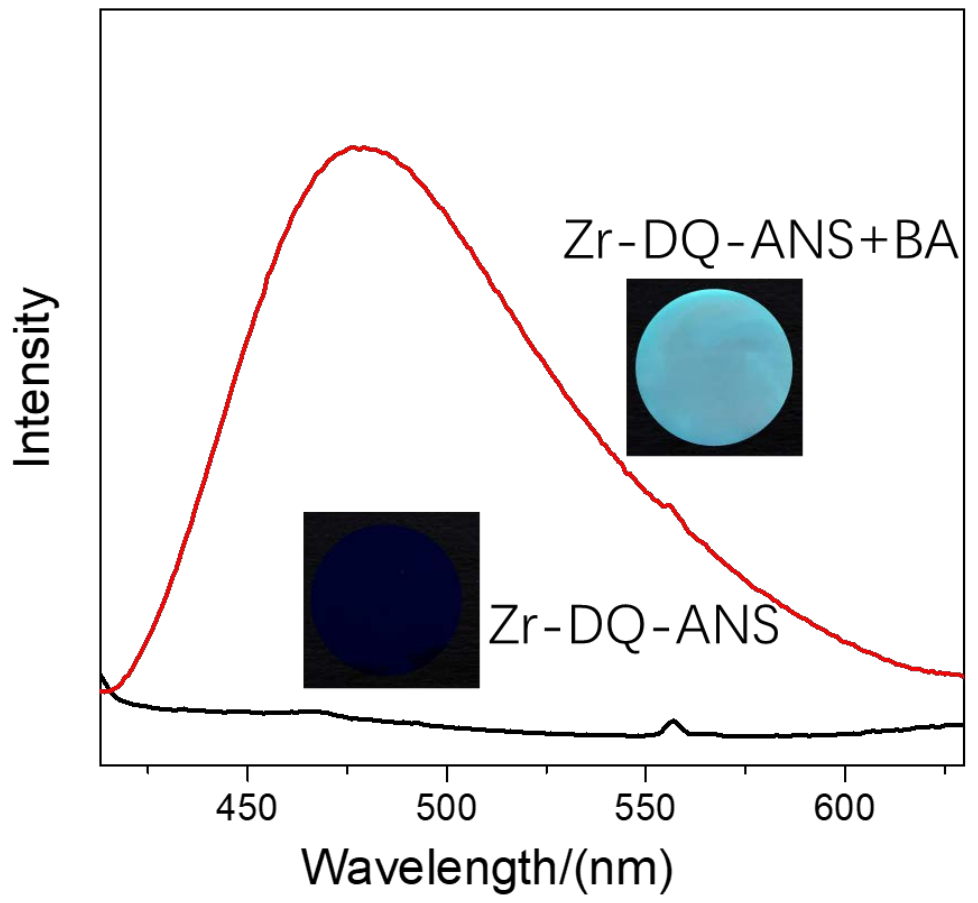

Figure S16. Solid-state emission spectra of Zr-DQ-ANS before (black) and after (blue) exposure to butylamine $(\mathrm{BA})\left(\lambda_{\mathrm{ex}}=377 \mathrm{~nm}\right)$. 


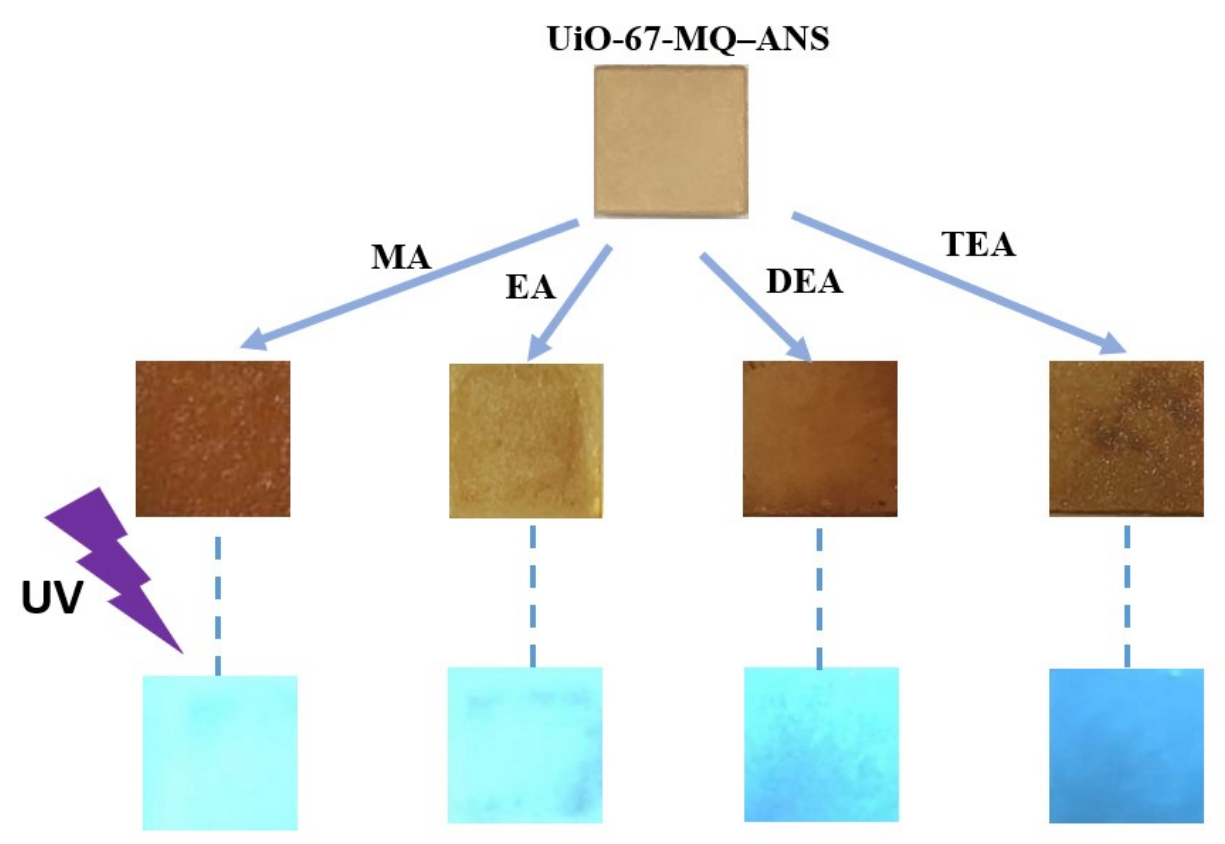

Figure S17. Photographs of the quartz slide supported Zr-MQ-ANS layers before and after treated with different amines: top and middle, under natural lights; bottom, under UV light $(365 \mathrm{~nm}) . \mathrm{MA}=$ methylamine, $\mathrm{EA}=$ ethylamine, $\mathrm{DEA}=$ diethylamine, $\mathrm{TEA}=$ triethylamine.

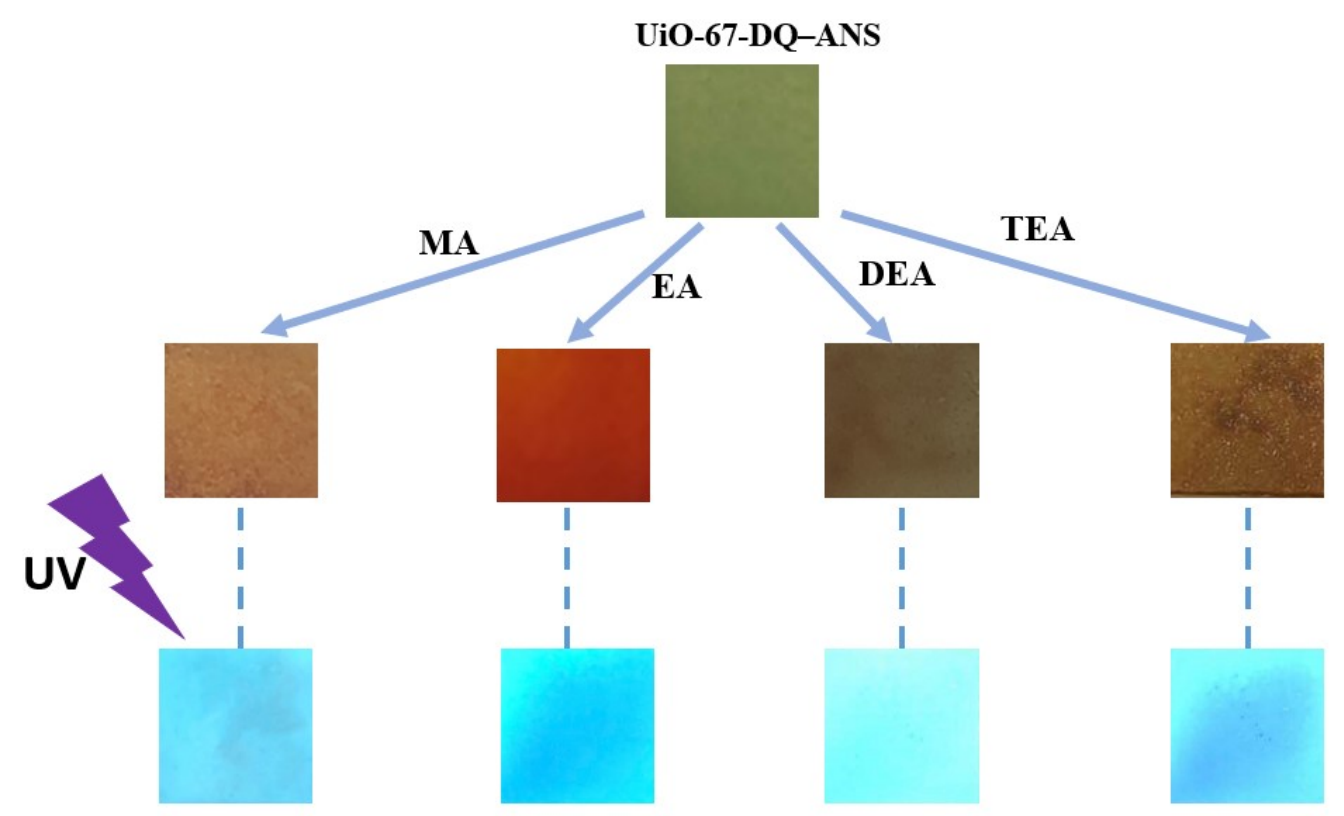

Figure S18. Photographs of Zr-DQ-ANS encounters different amines resulting in color changes and fluorescence under UV light $(365 \mathrm{~nm})$. MA = methylamine, EA = ethylamine, DEA = diethylamine, $\mathrm{TEA}=$ triethylamine. 